\title{
Mr. T.: Automated Electron Cryotomography for JEOL 2010F TEM
}

\author{
Chang J*, Marsh $\mathrm{M}^{*}$, Rixon $\mathrm{F}^{* *}$, and Chiu $\mathrm{W}^{*}$
}

*Program in Structural and Computational Biology and Molecular Biophysics, and National Center for Macromolecular Imaging, Baylor College of Medicine, Houston, TX 77030, USA. **MRC Virology Unit, Institute of Virology, Glasgow G11 5JR, United Kingdom

Electron cryotomography is one method for examining the structure of biological specimens in their native state without the perturbation associated with sectioning or chemical fixatives [1-4]. The technique relies on multiple exposures of the same specimen area over a wide range of tilt angles, and, consequently, is time-consuming and tedious even for an expert microscopist. We have developed a software package called Mr. T. to facilitate acquisition of low-dose tilt-series even by inexperienced users. Mr. T. runs on JEOL 2010F microscope equipped with JEOL's 5Axis FasTEM and a Gatan 4kx4k CCD camera. The single-particle imaging package JAMES, developed at the NCMI [5], integrates control of the microscope and CCD camera. We designed Mr. T. to reuse the JAMES libraries for instrument control. The result is a simple graphical user interface (GUI) that improves data quality and throughput, allowing for routine tilt-series data collection on a JEOL 2010F cryomicroscope.

Mr. T. is designed to image the same feature-of-interest for multiple exposures over a userdirected range of tilt angles while minimizing dose on the specimen. An essential part of acquiring a tilt-series is compensating for goniometer imperfections as the cryostage passes through a wide range of tilt angles. Without accurate compensation, the feature-of-interest is not maintained in the field-of-view over the course of the tilt-series. Mr. T. solves the featuretracking task with both automated and manual methods. Automated tracking relies on crosscorrelation between successive images to assure that any X-Y displacement between the two images is compensated for in the following image by the deflectors. Manual tracking is an interactive process that is used for specimens where correlation-based tracking is likely to fail. With manual tracking, after each image is acquired the user selects the center of the feature-ofinterest using the GUI (Fig. 1B); the deflectors are then adjusted so that in the next exposure the feature-of-interest is re-centered.

It is essential to distribute the dose over the tilt-series, often as many as 140 images, without overexposing the sample [6]. Mr. T. allows the user to specify the total dose for the tilt-series, as well as the initial and final tilt angles, and the angular increment (either constant or Saxton). From these input parameters, Mr. T. computes a tilt-plan containing a list of planned exposures including the angle and the exposure time for each exposure. Exposure time can be distributed evenly or secant-weighted to compensate for different ice thickness at different tilts. Furthermore, the user can adjust the tilt-plan by adding, deleting, or editing the angle and exposure time for any planned exposure (Fig. 1A).

At this time Mr. T. implements the features required for routine low-dose tomographic imaging of ice-embedded herpes capsid particles (Fig. 2). Several other specimens including whole cell and lipid vesicles have been imaged with this system. 3-D tomograms have been reconstructed using the EMAN software. The software feature set in Mr. T. continues to grow as we continue 
development, including automatic focusing routines and batch acquisition of multiple tomograms automatically.

\section{References}

[1] W. Baumeister et al. TRENDS Cell Biol 9 (1999) 81.

[2] A.J. Koster et al. J Struct Biol 120 (1997) 276.

[3] R. McIntosh et al. TRENDS Cell Biol 15 (2005) 43.

[4] B.K. Rath et al. J Struct Biol 120 (1997) 210.

[5] C.R. Booth et al. J Struct Biol 147 (2004) 116.

[6] B.F. McEwen et al. J Struct Biol. 138 (2002) 47.

[7] This research was supported by NCRR (P41RR02250), NIAID (R01AI38469), and the R Welch Foundation.
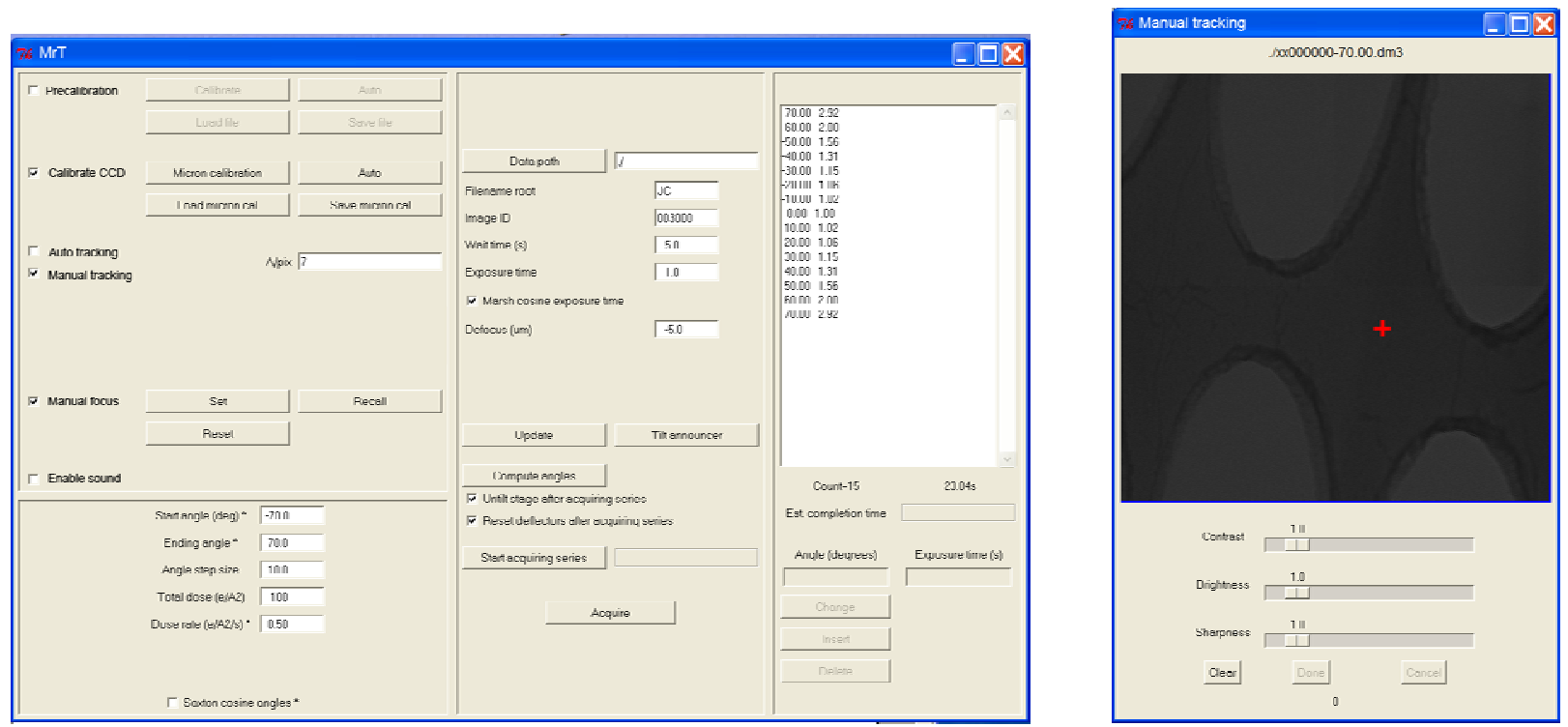

FIG. 1. Graphical user interface for Mr. T. (A) Tracking by precalibrating the stage, automatic, or manual can be selected, as well as focusing. The angle range, angle increment, and dose are set in the bottom left panel. The center panel contains the file path for the CCD images, file names, exposure time, and defocus. The right panel shows the angles and the exposure times, and allows users to add or modify arbitrary angles and exposures. (B) The manual tracking GUI shows the image of a Quantifoil grid that was just acquired. The user selects the center of interest (red cross) for the next image with a mouse click.

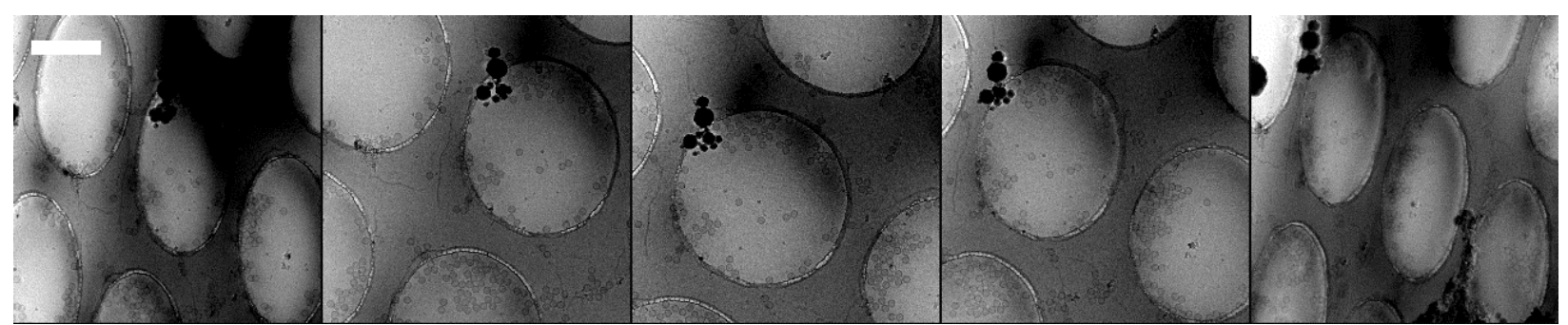

FIG. 2. Examples in a tilt series of 65 images $\left(-64^{\circ}\right.$ to $+64^{\circ}$ at $2^{\circ}$ increments) of Herpes capsid particles embedded in ice acquired with Mr. T. at $13800 \times$ in the JEOL $2010 \mathrm{~F}$ cryomicroscope onto a Gatan $4 \mathrm{k}$ CCD camera. Shown are the images from $-60^{\circ},-30^{\circ}, 0^{\circ}, 30^{\circ}$, and $60^{\circ}$. Bar is $1 \mu \mathrm{m}$. 